\section{Spatiotemporal chaos}

SIR - Egolf and Greenside ${ }^{1}$ recently investigated the relation between dimension density and correlation length in an extended spatiotemporally chaotic system. For spatiotemporally chaotic systems the fractal dimension, $D$, of the attractor typically grows proportionally to the volume of the system, making the dimension density $\mathrm{d} D / \mathrm{d} V$ a more appropriate quantity. The reason is that the chaotic fluctuations have a finite correlation length $\xi$, so distant parts behave almost independently. Assuming that $\xi$ is the only important length scale, it is natural to expect the dimension density times the correlation volume (the total number of degrees of freedom inside each correlation volume) to be constant. In other words (for a one-dimensional system)

$$
\mathrm{d} D / \mathrm{d} L \approx 1 / \xi
$$

Egolf and Greenside ${ }^{1}$ computed dimension densities and correlations for the complex Ginzburg-Landau equation

$\partial_{t} u(x, t)=u+\left(1+i c_{1}\right) \partial_{x}^{2} u-\left(1-i c_{3}\right)|u|^{2} u$

which describes the dynamics of a complex field $u=r e^{i \phi}$ in a one-dimensional medium. (This equation can, for example, describe an oscillatory chemical reaction; the complex amplitude $u$ is the Fourier component corresponding to the temporal period.)

The simulations were carried out in a regime where it is believed ${ }^{2}$ that the nature of the chaotic dynamics changes from 'defect' to 'phase' turbulence. Approaching the transition from the defect turbulence side, the correlation length $\xi$ was found to grow rapidly, and thus one should expect equation (1) to hold, but this turned out not to be so. The rapid variations in $\xi$ are not reflected in $\mathrm{d} D / \mathrm{d} L$. This discrepancy was left by Egolf and Greenside as a provoking open question .

To understand what goes wrong, one has to understand the nature of the transition occurring in equation (2). In the defect-dominated state, the phase of the complex field $u$ is not globally well defined, since it jumps by $\pm 2 \pi$ around each defect. In the phase-turbulent regime, however, the defects have little or no role. The phase field becomes continuous and is expected to be well approximated by the Kuramoto-Sivashinsky (KS) equation ${ }^{3}$ :

$$
\begin{gathered}
\partial_{t} \phi(x, t) \approx-v \partial_{x}^{2} \phi-\mu \partial^{4}{ }_{x} \phi-\lambda\left(\partial_{x} \phi\right)^{2}, \text { with } \\
v=\left(c_{1} c_{3}-1\right), \mu=1 / 2 c_{1}{ }^{2}\left(1+c_{3}{ }^{2}\right) \text { and } \\
\lambda=\left(c_{1}+c_{3}\right)
\end{gathered}
$$

(although note that the nonlinear terms which are left out can effectively renormalize these values). The linear instability of the KS equation creates a cell structure with typical cell sizes $l_{c}=2 \pi \sqrt{ } 2 \alpha$ with $\alpha=\sqrt{ } \mu / \nu$ and from the work of Manneville ${ }^{4}$ the dimension density is known to be close to 2 per cell, or $\mathrm{d} D / \mathrm{d} L \approx 0.23 / \alpha$, which, for the parameter values close to

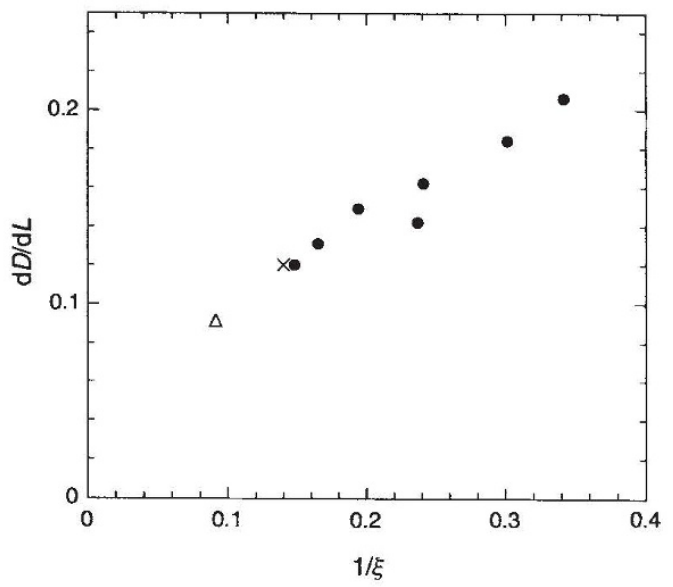

Dimension density $\mathrm{d} D / \mathrm{d} L$ against inverse information length $\xi^{-1}$ for the complex Ginzburg-Landau equation. Our simulations had $L=1,000$ and used a pseudospectral method. Information functions were computed from the evolution of $u(x, t)$ over $1,310,720$ time units. The values of $\mathrm{d} D / \mathrm{d} L$ are from ref. 1; the points with $\mathrm{d} D / \mathrm{d} L<0.15$ are in the phase chaos regime; those with $\mathrm{d} D / \mathrm{d} L>0.15$ correspond to defect chaos. See text for discussion of symbols.

the transition studied by Egolf and Greenside, is of the same order as the dimension density for the complex Ginzburg-Landau equation. Thus the cellular phase structure should introduce a new relevant length scale of the order of $l_{c}$. Further, equation (1) is trivially satisfied if we take $l_{c}$ as the correlation length $\xi$. This can be seen by rescaling $x=\alpha x^{\prime}, t=\beta t^{\prime}$ and $\phi=$ $\gamma \phi^{\prime}$ with $\alpha=\sqrt{ } \mu / v, \beta=\mu / v^{2}$, and $\gamma=v / \lambda$ which makes all the coefficients $\nu, \mu$ and $\lambda$ unity.

The correlation function studied in ref. 1 is $C\left(x-x^{\prime}\right)=\left\langle u(x, t) u^{*}\left(\mathrm{x}^{\prime}, t\right)\right\rangle$. This function is appropriate for the defectdominated phase, but rather insensitive to the phase fluctuations on the scale $l_{c}$. From correlations of $r(x, t)$, and of $\partial_{x} \phi$ $(x, t)$ in the phase turbulence regime, we can pick out a shorter length scale of the order of $l_{c}$. To avoid the troubles connected with oscillations of standard correlation functions, whose exponential decay is seldom very convincing in spatiotemporal chaos, we use so-called information functions $^{5,6}$, which express the correlations between the fields $A(x, t)$ and $A\left(x^{\prime}, t\right)$ at two spatially separated points directly in terms of their joint probability distribution function. We find simple exponential decay of these functions, and this method allows us to compute coherence lengths $\xi$ of the order of, or even below, $l_{c}$.
In the figure we show the dimension densities $\mathrm{d} D / \mathrm{d} L$ from ref. 1 against our coherence length $\xi$. The dots correspond to information functions for the modulus $r$, varying $c_{3}$ (from left to right) between 0.7 and 0.9 with $c_{1}$, fixed at 3.5. The cross and the triangle are found from information functions for $\partial_{x} \phi$ for the full complex Ginzburg-Landau equation and for the KS approximation, respectively, both at $c_{3}=0.7$. Note the closeness of all three quantities in the phase-turbulent regime $\left(c_{3}=0.7\right)$, where they are of the order $l_{c} / 2$. In the KS approximation, both $\xi$ and $(\mathrm{d} D / \mathrm{d} L)^{-1}$ scale as $\alpha$. Thus the plot for varying $c_{3}$ would be a straight line from the origin through the triangle, with equation (1) obviously satisfied in that approximation.

In contrast, the length scale computed from ref. $1, C\left(x-x^{\prime}\right)=$ $\left\langle u(x, t) u^{*}\left(x^{\prime}, t\right)\right\rangle$, should not satisfy equation (1). In the KS approximation, this corresponds to computing the correlation function of $u(x, t)=$ const $\times e^{i \phi(x, t)}$, for which (following ref. 2) the resulting correlation length $\xi_{1}$ is expected to scale as $\alpha / \gamma^{2}$, being sensitive to scaling of the phase field. Thus, again in the KS approximation, which should become better as $c_{3}$ is lowered into the phase-turbulent regime, the product $\mathrm{d} D / \mathrm{d} L \cdot \xi_{1} \rightarrow \infty$ as the Benjamin-Feir transition $\left(c_{3}=1 / c_{1}\right)$ is approached.

Although we do not claim to have found a general recipe for computing correlations of the chaotic fluctuations, we believe that we have pointed to the relevant length scales in the present problem for which there is no contradiction with equation (1).

We have benefited from discussions with Henry Greenside during this work.

\section{Tomas Bohr}

The Niels Bohr Institute,

Blegdamsvej 17,

2100 Copenhagen $\varnothing$,

Denmark

\section{Eric Bosch}

Willem van de Water

Physics Department,

Eindhoven University of Technology,

PO Box 513,

$5600 \mathrm{MB}$, Eindhoven,

The Netherlands

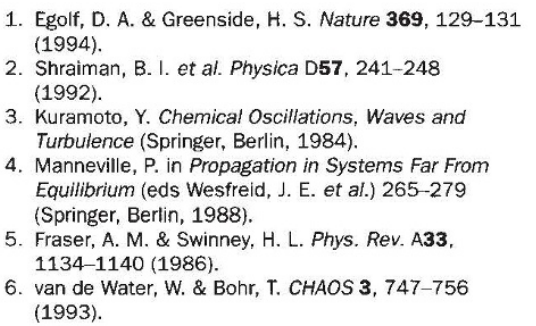

